\title{
Enfrentamento e Resiliência Familiar na Tomada de Decisão pela Gastrostomia Infantil
}

\author{
Natália Ferraz Novaes ${ }^{1}$ \\ ${ }^{1}$ Universidade Federal do Rio de Janeiro, RJ, Brasil.
}

\author{
Ana Cristina Barros Cunha ${ }^{1}$ \\ ${ }^{1}$ Universidade Federal do Rio de Janeiro, RJ, Brasil.
}

Resumo: A indicação do procedimento de gastrostomia em uma criança reverbera em suas famílias como um momento de crise, com a ruptura do padrão de funcionamento familiar. A decisão pela gastrostomia infantil envolve crenças e valores familiares, além de aspectos psicológicos, como o enfrentamento e a resiliência familiar. O objetivo deste estudo foi investigar o enfrentamento e a resiliência familiar de cuidadores de crianças frente à tomada de decisão pela gastrostomia infantil. Quatro cuidadores principais de crianças com indicação para gastrostomia responderam ao Inventário COPE, para avaliar estratégias e estilos de enfrentamento, e a entrevista "Indicadores de Resiliência Familiar", elaborada para esse estudo. O relato verbal dos cuidadores foi analisado com base nos indicadores de resiliência familiar proposto por Walsh, adotando-se a Análise de conteúdo de Bardin. Os indicadores de resiliência familiar encontrados foram a "Busca em extrair significado da adversidade" e a "Perspectiva positiva", junto com um enfrentamento caracterizado por maior frequência de estratégias como "Coping ativo", "Planejamento", "Reinterpretação positiva e crescimento", e "Retorno para a religiosidade". Esses achados sugerem que as famílias foram capazes de superar os desafios impostos pela gastrostomia infantil e enfrentar as adversidades de forma ativa e planejada, quando reinterpretaram sua experiência atribuindo significados positivos à situação. Concluímos que a tomada de decisão resiliente e positiva pauta-se na negociação dos valores familiares e o saber clínico da equipe de saúde. Destacamos o trabalho empático dos profissionais de saúde com os cuidadores, fortalecendo as potencialidades destes para a sobrevivência e superação dos desafios impostos pela gastrostomia.

Palavras-chave: Criança Hospitalizada. Cuidadores. Coping. Resiliência. 


\title{
Coping and Family Resilience in Decision-Making about Children's Gastrostomy
}

\begin{abstract}
The indication of the gastrostomy procedure for children is a moment of crisis for their families, with the rupture of the pattern of family functioning. The decision to perform a gastrostomy involves beliefs and family values, as well as psychological aspects such as coping and family resilience. The aim of this study was to investigate the coping and family resilience of caregivers who decide that their children will have infant gastrostomy. Four main caregivers of children with indication for a gastrostomy answered the COPE Inventory, to evaluate coping strategies and styles, and were interviewed using the interview entitled "Indicators of Family Resilience", elaborated for this study. The verbal report of caregivers was analyzed using the indicators of familiar resilience proposed by Walsh, adopting the Bardin Content Analysis. The most frequent family resilience indicators were the "Questioning to extract meaning from adversity" and the "Positive perspective", along with coping strategies characterized by a high frequency of the use of "Active Coping", "Planning", "Positive Reinterpretation and Growth", and "Return to religion". These findings suggest that families were able to overcome the challenge posed by the infant gastrostomy, and to cope with the adversity in an active and planned way when they reinterpret the experience and attribute a new positive meaning to the situation. We conclude that the resilient and positive decisionmaking is based on the negotiation of family values and knowledge of the health team. We highlight the empathic work of health professionals with caregivers, strengthening the potential of these to survive and overcome the challenges posed by gastrotomy.
\end{abstract}

Keywords: Hospitalized Child. Caregivers. Coping. Resilience.

\section{Enfrentamiento y Resistencia en la Toma de Decisiones por Gastrostomía para Niños}

Resumen: La indicación del procedimiento de gastrostomía en un niño reverbera en sus familias como un momento de crisis, con la ruptura del funcionamiento familiar. La decisión de la gastrostomía implica creencias y valores familiares, así como aspectos psicológicos como el enfrentamiento y la resiliencia familiar. El objetivo de este estudio fue investigar la capacidad de adaptación y la resistencia familiar de los cuidadores de niños en relación con la toma de decisiones por la gastrostomía infantil. Cuatro cuidadores principales de niños remitidos para gastrostomía respondieron al Inventario COPE para evaluar las estrategias y estilos de enfrentamiento, y la entrevista "Indicadores de resiliencia familiar" diseñada para este estudio. El informe verbal de los cuidadores se analizó en función de los indicadores de resiliencia familiar propuestos por Walsh, utilizando el Análisis de contenido de Bardin. Los indicadores de resistencia familiar encontrados fueron la "Búsqueda en la extracción de significado de la adversidad" y la "Perspectiva positiva", junto con una confrontación caracterizada por una mayor frecuencia de estrategias como "Coping activo", "Planificación”, “Reinterpretación positiva y crecimiento", y "Regreso a la religiosidad”. Estos resultados sugieren que las familias pudieron superar los desafíos planteados por la gastrostomía infantil y enfrentar la adversidad de manera activa y planificada cuando reinterpretaron su experiencia asignando significados positivos a la situación. Concluimos que la toma de decisiones resistentes y positivas se basa en la negociación de los valores familiares y el conocimiento clínico del equipo de salud. Destacamos el trabajo empático de los profesionales de la salud con los cuidadores, fortaleciendo su potencial de supervivencia y superando los desafíos impuestos por la gastrostomía. Palabras clave: Niño Hospitalizado. Cuidadores. Coping. Resistencia. 


\section{Introdução}

Segundo a Portaria no 400 de 16 de novembro de 2009 (Portaria No 400, 2009), que estabelece diretrizes nacionais para a atenção à saúde das pessoas ostomizadas, define-se a gastrostomia (GTT) como um procedimento cirúrgico que visa realizar a comunicação do estômago ao meio exterior. De acordo com este documento, a ostomia é um procedimento cirúrgico que consiste na exteriorização dos sistemas digestório, respiratório ou urinário, sendo o estoma a abertura artificial entre os órgãos internos com o meio externo. Neste sentido, a gastrostomia é um estoma que funcionaria como via suplementar de alimentação.

Vários estudos (Anselmo, Tercioti Junior, Lopes, Coelho Neto, \& Andreollo, 2013; Santos et al., 2011; Souza, 2016) discutem o que leva à indicação de uma GTT e sinalizam que a alimentação enteral, por sondas nasal ou oral, e a GTT devem ser adotadas diante da necessidade de suporte alimentar para melhora do estado nutricional do paciente "por ser mais confortável, permitir maior mobilidade do paciente, não interferir com a respiração e os mecanismos fisiológicos de limpeza das vias aéreas" (Santos et al., 2011, p. 39). A GTT é indicada para pacientes com distúrbio motor severo de deglutição (Souza, 2016) ou ainda pacientes com trato intestinal intacto, mas impossibilitados de alimentar-se pela via oral (Anselmo et al., 2013). No caso da GTT infantil, é indicado o procedimento com tubos orogástricos ou nasogástricos para crianças com paralisia cerebral, por exemplo, para prevenir riscos de otites, sinusites, ulcerações esofágicas, perfurações intestinais ou gástricas, ainda que por curtos períodos, em geral, de no máximo seis semanas (Brasil, 2013).

Ressalta-se a escassez de dados de prevalência de crianças gastrostomizadas no Brasil, assim como do perfil desta população. De acordo com dados estatísticos do Instituto de Pediatria e Puericultura Martagão Gesteira da Universidade Federal do Rio de Janeiro (IPPMG/UFRJ), hospital universitário de referência em pediatria no Estado do Rio de Janeiro, são realizadas em média uma GTT infantil por mês e o tempo de internação para a realização da cirurgia e posterior adaptação é de aproximadamente 30 dias. Por sua vez, em estudo realizado com crianças com paralisia cerebral na cidade de Botucatu em São Paulo, Martins (2013) constatou que $92,00 \%$ dos pacientes fizeram gastrostomia cirúrgica e a maior indicação era a desnutrição, para 91,17\% dos casos.

\section{Valores e crenças familiares e o impacto psicológico da decisão pela gastrostomia}

A indicação de uma criança para o procedimento de GTT implica na inclusão e participação direta da sua família, tendo em vista a necessária autorização familiar para que o procedimento ocorra. Neste momento devem ser considerados os cuidados domiciliares no pós-alta, além dos valores, crenças e significados atribuídos pela família à alimentação, já que esta é permeada por valores socioculturais, quando o ato de comer pode ser representado como uma atividade social e fonte de vínculo familiar (Cruz, Angelo, \& Gamboa, 2012).

De acordo com Mahant, Jovcevska, e Cohen (2011), para alguns pais o processo de comer pela boca pode ser percebido como uma fonte de prazer agradável para a criança e a GTT representar uma negação deste prazer para a família; para outros pais, o tubo da GTT não é visto como algo natural, porque não corresponde à percepção de uma família normal. Segundo Mahant et al. (2016), a tomada de decisão pela alimentação via GTT é um momento de conflito e período de incertezas e grande estresse para os pais, quando problemas de alimentação desafiam as narrativas culturais da família do que significa ser bons pais e limitam a participação da família e seus membros na vida social. Sentimentos como desapontamento e incapacidade são relatados pelos pais no momento da tomada de decisão pela gastrostomia, junto com o medo de que seus filhos sejam estigmatizados e consequentemente marginalizados, isolados e discriminados (Cruz et al., 2012).

Portanto, a indicação de GTT em crianças reverbera na família como um momento de crise, no qual sua estrutura e dinâmica de organização familiar são abaladas, assim como a idealização daquele filho. Franco (2016), em estudo com pais de crianças com transtornos graves do desenvolvimento, afirma que a idealização do filho, ou seja, a representação mental e afetiva de um bebê ideal é fundamental para a construção da parentalidade, pois permite que os pais cuidem do seu filho real e desenvolvam um vínculo afetivo com ele. Logo, o diagnóstico de uma patologia severa representa a perda desta idealização, que encerra a perda de esperanças e expectativas, sendo inevitável que ocorram sentimentos depressivos nos familiares (Franco, 2016).

O problema central que se coloca neste momento da decisão pela GTT é como esses pais poderão "alcan- 
çar um novo nível de funcionamento depois da crise, tornando-se pais $\backslash$ mães amorosos e nutrientes e não apenas funcionais e utilitários para um filho que não corresponde à sua idealização" (Franco, 2016, p. 38). Nesse sentido, um procedimento como a GTT pode representar para a família a perda de uma perspectiva de normalidade do filho, com impacto psicológico que repercute na quebra das expectativas de uma vida normal e saudável. A gastrostomia será vista, então, como uma marca de deficiência, a qual resulta em um estigma que atravessa a ligação afetiva entre filhos e pais e coloca esses últimos em conflito com sua capacidade de serem bons pais e decidirem o que é melhor para seus filhos.

Portanto, estudar como se caracteriza o enfrentamento dos cuidadores frente a decisão pela gastrostomia da criança, reconhecendo os valores, atitudes e crenças familiares que pautam a resiliência da família para enfrentar esse momento tão difícil da decisão pela GTT de um filho, é imprescindível para se compreender quais os recursos as famílias adotam para lidar com os conflitos típicos desse momento.

\section{Estratégias de enfrentamento e resiliência familiar}

Considerando que este trabalho pretende investigar o enfrentamento das famílias no momento de tomada de decisão pela GTT com base na investigação de estratégias de enfrentamento (coping) e indicativos de resiliência familiar, faz-se importante apresentar estes dois conceitos. Entende-se por resiliência a capacidade de se reestruturar mais fortalecido e com mais recursos diante das adversidades (Walsh, 2005). Para Walsh (2005), a resiliência é um processo ativo que está para além de sobreviver aos desafios e que engloba resistir, reestruturar e crescer frente às crises. De acordo com a perspectiva dessa autora, a resiliência familiar pode ser compreendida a partir de três domínios do funcionamento familiar: 1) "Sistema de crenças"; 2) "Processos organizacionais"; e 3) "Processos de comunicação”. Estes três domínios da resiliência familiar constituem a estrutura necessária para identificação das potencialidades e vulnerabilidades de uma família (Walsh, 2005).

O "Sistema de crenças" é um domínio central do funcionamento familiar, que abrange valores, convicções e atitudes que desencadeiam reações emocionais, decisões e ações. O sistema de crenças pode ser considerado um indicador de resiliência familiar quando as crises são enfrentadas extraindo-se significados da experiência vivida, vinculando-a ao mundo social, às crenças culturais e religiosas, ao passado multigeracional e aos planos futuros. Segundo Walsh (2005), essas crenças compreendem a capacidade de extrair significado da adversidade, de adotar uma perspectiva positiva e de estabelecer crenças transcendentes para os valores e os propósitos da vida que servem como consolo e conforto. $\mathrm{O}$ modo como as famílias encaram os desafios faz diferença no enfrentamento e no manejo e controle de uma determinada situação, assim como na disfunção e no desespero experimentado pelos familiares diante da situação de crise.

Já os "Processos organizacionais" se caracterizam pela maneira com as famílias mobilizam recursos sociais e econômicos, resistem às crises e se reorganizam para lidar de maneira eficiente com a adversidade. Os processos organizacionais fundamentais para a resiliência familiar são: flexibilidade, conexão e recursos sociais e econômicos. A flexibilidade diz respeito ao desenvolvimento de uma estrutura flexível, porém estável, necessária para o bom funcionamento das famílias. A conexão é definida como o equilíbrio entre unidade, apoio mútuo e colaboração com a separação e a autonomia dos indivíduos. Os recursos sociais e econômicos estão relacionados aos vínculos sociais e profissionais, ou seja, a família ampliada, a rede social e de equilíbrio entre os sistemas familiar e profissional. Portanto, os padrões organizacionais familiares são mantidos por normas externas e internas, reforçadas pelos sistemas cultural e de crenças familiares.

Por fim, os "Processos de comunicação" são definidos com base em duas funções: o aspecto do "conteúdo", transmissão de fatos, opiniões ou sentimentos acerca da situação; e o aspecto do "relacionamento", que define a natureza do relacionamento, ou seja, o posicionamento do indivíduo dentro da sua estrutura familiar. Trata-se de um indicador de resiliência porque, para Walsh (2005), a comunicação facilita o funcionamento familiar. Em momentos de crise é preciso esclarecer as situações estressantes, pois as experiências tornam-se mais compreensíveis e administráveis quando as mensagens são compartilhadas e discutidas. Portanto, intervenções que visem fortalecer a resiliência familiar se focam no aumento da competência comunicacional dos membros da família para se expressarem e reagirem às necessidades $\mathrm{e}$ 
preocupações com base, por exemplo, na negociação de mudanças no sistema familiar para responder às novas demandas da situação de crise (Walsh, 2005).

Levantamento da produção bibliográfica mundial sobre resiliência psicológica no período de 1997 a 2004, feito por Souza e Cerveny (2006), destacou que dentre o total de 307 referências, somente 40 se centravam no estudo da resiliência familiar no contexto da saúde e apenas sete delas eram nacionais, o que confere relevância a este estudo. Esses autores citam ainda que, dentre as abordagens teóricas que estudam resiliência familiar, a proposta de Walsh e a de McCubbin e colaboradores se destacam, por reconhecerem o caráter sistêmico e ecológico como importante nestes estudos. Para Yunes (2003), a partir do final dos anos 1980, com as pesquisas sobre o coping, as competências e a adaptação de famílias, se iniciaram os estudos sobre resiliência familiar na tentativa de ultrapassar um enfoque individual para propor um enfoque sistêmico, com base na investigação das redes de suporte social e das crenças construídas socialmente pelas famílias para o enfrentamento das adversidades. Assim sendo, buscou-se articular o presente estudo sobre resiliência familiar com o conceito de enfrentamento ou coping por entender que estes fenômenos estão intimamente ligados à vivência da família diante da crise deflagrada frente à tomada de decisão pela GTT infantil.

Entende-se por coping um conjunto de estratégias, cognitivas e comportamentais, utilizadas pelas pessoas para lidarem com a situação de estresse e se adaptarem em circunstâncias adversas (Antoniazzi, Dell'Aglio, \& Bandeira, 1998). Segundo Carver, Scheier e Weintraub (1989), os estilos de coping podem refletir uma tendência a responder de uma forma particular, quando a pessoa é confrontada com uma série específica de circunstâncias geradoras de estresse. Portanto, "estilos de coping" é um conceito proposto pela Teoria cognitiva disposicional do coping desenvolvida por Carver et al. (1989) que, diferentemente das estratégias de enfrentamento, representam formas habituais ou tendências individuais para lidar com estresse e se relacionam às características de personalidade e às capacidades autorregulatórias (Carver et al., 1989; Carver, \& Connor-Smith, 2010; Carver, \& Scheier, 2011). Trata-se de uma tendência particular de responder ao estressor, ou seja, de adotar estratégias comportamentais e emocionais específicas usando a autorregulação diante da situação de estresse (Carver et al., 1989; Carver, \& Connor-Smith, 2010; Carver, \& Scheier, 2011; Cravinho, \& Cunha, 2015).

Nessa perspectiva teórica do coping, a partir do estudo dos aspectos psicodinâmicos relacionados ao desenvolvimento, a personalidade tem forte influência sobre processos desenvolvimentais como o enfrentamento ou o coping (Carver et al., 1989; Carver, \& Connor-Smith, 2010; Carver, \& Scheier, 2011). Mais especificamente, para Carver et al, (1989), partindo dos estudos de Lazarus e Folkman (1989), o coping pode focar no problema, na emoção, mas também reunir ambos os esforços, sempre com um caráter disposicional por envolver mecanismos de autorregulação pautados em características de temperamento e personalidade. Para tanto, no coping focalizado no problema a pessoa dirige esforços para lidar com o estressor através de atitudes que visem solucionar ou minimizar diretamente o seu impacto; enquanto que no coping focalizado na emoção, ela busca minimizar o sofrimento trazido pelo estressor através de ações que envolvem o manejo das emoções, como a busca de suporte emocional e regulação emocional. Ambas as respostas de coping possuem finalidades distintas e se baseiam na interpretação cognitiva que o indivíduo elabora do evento estressor, sempre como um desafio, uma ameaça ou um dano ao seu self que exige esforços de autorregulação, ou seja, capacidades biologicamente e psicologicamente adquiridas responsáveis pela modulação das emoções e comportamentos para atingir uma meta (Carver et al., 1989; Carver, \& Connor-Smith, 2010; Carver, \& Scheier, 2011).

Em síntese, estratégias de enfrentamento focalizadas no problema e na emoção se complementam promovendo ora estratégias eficazes ou um estilo de coping engajado, o "coping engagement", que pressupõem a ideia de compromisso e envolvimento da pessoa com seu processo de enfrentamento pelo uso de estratégias ativas de coping (busca de apoio, regulação emocional, aceitação e reestruturação cognitiva); ora estratégias pouco eficazes ou um estilo de coping desengajado, o "disengagement coping", que pressupõem o uso de estratégias como evitação, negação e ilusão, baseando-se em esforços para ignorar o estressor ou inibir reações comportamentais e emocionais, por vezes sob uma perspectiva fantasiosa (Carver, \& Connor-Smith, 2010). Ambos os estilos de coping representam tendências de se autorregular e enfrentar os estressores e as adversidades da 
vida (Carver et al., 1989; Carver, \& Connor-Smith, 2010; Carver, \& Scheier, 2011).

Tendo em vista que o auxílio às famílias na tomada de decisão pela GTT deve basear-se nas potencialidades dos cuidadores para lidar com a crise, adaptar-se ao futuro imediato e, em longo prazo, reorganizar a vida (Walsh, 2005), reconhece-se como importante traçar algumas considerações sobre a função da equipe de saúde como facilitadora de um enfrentamento familiar resiliente frente às demandas inerentes à crise resultante da GTT.

\section{A equipe como facilitadora na tomada de decisão familiar}

Diante da representação simbólica que a alimentação pode ter para uma família e a crise inerente ao momento de tomada de decisão pela GTT, é possível pensar que a equipe de saúde pode assumir um papel importante no auxílio aos cuidadores para lidar com este momento. Vários estudos consideram importante propostas de intervenção baseadas na empatia da equipe para com a família, com protocolos de assistência baseados na educação, informação, suporte de alta qualidade e interação entre as famílias candidatas à GTT e aquelas que já passaram pelo processo (Franco, 2016; Mahant et al., 2011; Martínez-Costa et al.,2011; Shelton, \& Jackson, 2011; Pemberton, Frankfurter, Bailey, Jones, \& Walton, 2013; Schweitzer et al., 2014). As famílias têm potencialidades para enfrentar períodos de desafio, as quais podem ser estimuladas pela equipe por uma postura empática que compreenda os valores e crenças familiares e desenvolva um trabalho que as ajude a usarem estas potencialidades no enfrentamento da crise e na elaboração dos sentimentos negativos inerentes à situação.

Nelson e Mahant (2014) propõem, como forma de intervenção familiar para cuidadores de crianças com deficiência neurológica grave, a chamada estratégia Shared Decision-Making (SDM). Essa estratégia consiste em traçar um plano de tratamento baseado nos valores familiares e nas evidências clínicas que a equipe de saúde obtém sobre cada caso. No SDM, os membros da família expressam seus objetivos e valores relacionados à decisão pela tecnologia de apoio e a equipe discute sobre os riscos e benefícios de cada uma das opções de intervenção para que o plano seja negociado em conjunto entre equipe e família na busca das melhores opções disponíveis.
Portanto, nem a equipe nem a família tem uma responsabilidade independente pela decisão; ao contrário, todos os lados são ouvidos e juntos decidem como prosseguir.

Para Schweitzer et al. (2014), um protocolo de educação pode resultar na melhoria dos resultados dos pacientes, no aumento do conhecimento do cuidador e na diminuição da ansiedade dele, o qual passa a ser mais bem aceito pela equipe de saúde. Em concordância com a ideia de uma intervenção visando a educação adequada dos cuidadores, Matínez-Costa et al. (2011) afirmam que a maioria dos pais ou tutores teriam aceitado melhor a GTT se anteriormente tivessem sido mais bem informados sobre os seus benefícios. Por outro lado, Cruz et al. (2012) apontam que as famílias vivenciam experiências negativas na comunicação devido à falta de informação ou devido a informações inadequadas, quando, na abordagem clínica, a equipe escolhe o procedimento sem considerar a experiência de vida das famílias. As famílias acreditam, assim, que os profissionais as fazem sentirem-se culpadas e envergonhadas pela condição nutricional da criança e não respeitam o esforço empreendido por elas para manter uma alimentação oral, além de, serem vistas como "más" pela equipe quando não concordam com a indicação da GTT (Cruz et al., 2012).

É fato que profissionais de saúde e pais têm percepções diferentes durante o processo de tomada de decisão pela GTT, sendo importante a informação e o suporte de alta qualidade para as famílias a fim de serem encorajadas a tomarem sua decisão. Em alguns casos, inclusive, a aceitação do procedimento de GTT foi significativamente retardada e com consequente deterioração nutricional da criança, o que poderia ser minimizado se houvesse uma intervenção baseada na cooperação entre os pais candidatos à GTT e pais de crianças que já realizaram o procedimento, recurso indicado como extremamente prático para evitar atrasos na aceitação da GTT (Matínez-Costa et al., 2011).

Muitos estudos apontam ainda para melhoria da qualidade de vida dos cuidadores após a realização da GTT; porém, poucos métodos de intervenção são propostos para o momento anterior ao procedimento (Avitsland et al., 2012; Martínez-Costa, Calderón, Pedrón-Giner, Borraz, \& Gómez-López, 2013; Pemberton et al., 2013; Susin, Bortolini, Sukiennik, Mancopes, \& Barbosa, 2012; Wilson, Gosche, Bishop, 
Liu, Moore, \& Nowicki, 2010; ). Susin et al. (2012), por exemplo, afirmam que as implicações do uso da GTT e os efeitos que ela produz nos familiares e cuidadores têm recebido pouca atenção dos pesquisadores, os quais se voltam mais para o estudo dos aspectos médicos e nutricionais. Especialmente no Brasil, não foram encontrados trabalhos que articulassem o enfrentamento e a resiliência familiar no estudo da tomada de decisão de cuidadores pela GTT infantil, ainda que autores como Noronha, Cardoso, Moraes e Centa (2009) considerem importante a investigação da resiliência em estudos sobre promoção da saúde de famílias. Para esses autores, profissionais de saúde devem buscar estratégias e recursos para fortalecerem a resiliência familiar e incluir isso no planejamento de suas ações de promoção de saúde, já que "as relações familiares funcionam como promoção da vida e bem-estar social, propiciando condições adequadas de vida, favorecendo o desenvolvimento das potencialidades de cada um e do grupo" (Noronha et al., 2009, p. 500).

Diante do exposto, estudos sobre os temas propostos são de relevância científica, porque se centram na preocupação em dar atenção e cuidar também dos cuidadores. Cruz et al. (2012) consideram que a percepção da família de que a cirurgia de GTT será bem-sucedida dependerá das circunstâncias pessoais dos familiares e da criança, incluindo o diagnóstico, o prognóstico e o suporte disponível aos membros da família e no sistema de saúde. Portanto, o enfrentamento da GTT infantil pela família é um fator de risco ao desenvolvimento infantil, pois, como afirma Franco (2016), quando os pais não estão disponíveis, emocionalmente, para decidirem de forma autônoma sobre a gastrostomia, eles questionam a importância desse procedimento para a evolução e saúde do filho. Desta forma, os pais, por se sentirem inseguros nas decisões relacionadas à sua função de cuidador, podem deixar de oferecer à criança aquilo que é o mais importante para ela: segurança, disponibilidade e envolvimento afetivos.

Considerando que a decisão pela GTT infantil envolve variáveis psicossociais, como as crenças e os valores familiares, os quais interferem na tomada de decisão pelo procedimento, e que conhecer os aspectos psicológicos inerentes a situação pode ajudar no auxílio aos cuidadores para o manejo e enfrentamento da questão, o objetivo desse estudo foi investigar o enfrentamento e a resiliência familiar de cuidadores de crianças com necessidades especiais de saúde frente à tomada de decisão pela gastrostomia infantil.

\section{Método}

Com base em delineamento descritivo qualitativo-quantitativo, a pesquisa contou com uma amostra de conveniência de quatro cuidadores de crianças com necessidades especiais de saúde, no caso Paralisia Cerebral e Síndrome de Down. Com idades que variavam entre 28 a 59 anos (média de 42 anos), dentre os cuidadores três eram mães (C2, C3, C4) e um era pai $\left(\mathrm{Cl}^{1}\right)$; somente este último trabalhava de forma remunerada. Ressalta-se que duas mães (C3, C4) afirmaram que precisaram parar de trabalhar para cuidar do filho. Metade deles tinha ensino médio completo e declararam-se casados, enquanto que os demais tinham ensino fundamental incompleto e declararam-se divorciados. Três cuidadores declararam ter mais de um filho (C1, C2, C3). Todos declararam ter crença espiritual $\backslash$ religiosa, tais como cristianismo (católico e evangélico).

Em todos os casos as crianças apresentavam desnutrição e dificuldade de alimentação por via oral. Todos os cuidadores eram os principais responsáveis pela criança e também pela decisão de autorizar ou não a realização da GTT. As crianças eram acompanhadas pelas equipes de Medicina, Nutrição, Psicologia, Fisioterapia e Serviço Social em um hospital infantil, ligado a uma Universidade Federal, localizado na cidade do Rio de Janeiro.

Pela rotina deste hospital é agendado mensalmente um procedimento de GTT; porém, nem sempre são realizados todos os procedimentos agendados em função das condições pré-operatórias da criança, que podem mudar e exigir até o cancelamento da cirurgia. Durante o período de realização desse estudo foram agendadas o total de quatro indicações de GTT e todos os cuidadores das crianças que seriam submetidas ao procedimento foram convidados. Não houve recusa de nenhum cuidador que foi abordado para esse estudo.

A coleta de dados foi realizada em sala da própria unidade hospitalar após aprovação pelo Comitê de Ética em Pesquisa da instituição (Número CAAE 58758116.4.0000.5264). Antes, porém, o projeto foi

\footnotetext{
${ }^{1}$ Os participantes serão identificados pela letra C (de Cuidador) seguido de um número de ordem da sua inclusão na amostra do estudo. Logo, C1 se trata do Cuidador 1, ou seja, o primeiro cuidador participante entrevistado para o estudo.
} 
apresentado aos profissionais do Setor de Psicologia, à Direção clínica do hospital e aos profissionais responsáveis pela unidade a fim de solicitar apoio para coleta e colaboração no recrutamento dos participantes. Após serem comunicados pela equipe de saúde sobre a indicação para a GTT, os participantes foram abordados, durante a internação da criança e convidados para o estudo com informações sobre a pesquisa e esclarecimentos sobre os objetivos e coleta. Os cuidadores foram entrevistados e responderam aos instrumentos de coleta de dados abaixo descritos, garantindo-se a total privacidade dos participantes e sua assinatura em um Termo de Consentimento Livre e Esclarecido (TCLE).

Para analisar as estratégias de enfrentamento e estilos de coping foi usado o Inventário COPE (Carver et al., 1989), que consiste em uma escala padronizada que identifica as estratégias de enfrentamento adotadas em situações de estresse. Para esse estudo foi usada uma versão adaptada para a realidade brasileira por Mazon, Carlotto e Câmara (2008) do Inventário COPE com o total de 60 itens com sistema de pontuação tipo Likert de 1 a 4 , sendo 1 para "não costumo fazer isso nunca" e 4 para "costumo fazer isso muito". A escala total se subdivide em 15 subescalas com os seguintes fatores: 1) coping ativo; 2) planejamento;3) supressão de atividades concomitantes; 4) coping moderado; 5) busca suporte social por razões instrumentais; 6) busca de suporte social por razões emocionais; 7) reinterpretação positiva; 8) aceitação; 9) religiosidade; 10) foco na expressão de emoções; 11) negação;12) desligamento comportamental; 13) desligamento mental;14) humor, e 15) uso de substâncias. A classificação ocorre por subescala através da soma dos valores de resposta a cada item, em que valores acima de 16 representam uso frequente ("sempre" ou "muitas vezes") do tipo de estratégia de coping, enquanto valores abaixo de 3 representam que a pessoa "nunca" usa aquele tipo de coping. Propõe-se o cálculo da mediana de cada tipo de EE para identificar a prevalência do tipo de coping para grupos (Carver et al., 1989).

Para analisar a resiliência familiar foi usado o roteiro de entrevista "Indicadores de Resiliência Familiar", elaborado especialmente para esse estudo com objetivo de coletar o relato verbal dos cuidadores que permitisse investigar e identificar os indicadores de resiliência familiar. O roteiro continha nove perguntas abertas, elaboradas com base nos três domí- nios de Resiliência Familiar propostos pela teoria de Walsh (2005), a saber: A) Sistema de crenças: 1- extrair significado da adversidade, 2- perspectiva positiva, 3- transcendência e espiritualidade; B) Processos organizacionais: 4- flexibilidade, 5- conexão, 6- recursos sociais e econômicos; e C) Processos de comunicação: 7- clareza, 8- expressão emocional aberta, 9- resolução colaborativa dos problemas.

A coleta de dados ocorreu em etapa única, com aplicação do Inventário COPE seguida da entrevista, que foi gravada e transcrita na íntegra. O processamento dos dados do Inventário COPE foi realizado de acordo com as normas propostas pelo instrumento e analisados quantitativamente em termos de medianas das estratégias de enfrentamento dos participantes, utilizando-se o software Microsoft Excell. Com base na metodologia de Análise de conteúdo de Bardin (2006), foi adotada uma análise categorial extraindo-se dos relatos verbais coletados nas entrevistas dados das falas dos participantes, que foram processados e analisados com base nas definições dos domínios de Resiliência Familiar proposto por Walsh (2005) anteriormente descritas.

Adotando-se o referencial teórico já mencionado, todos os dados foram analisados qualitativamente buscando relações entre os dados quantitativos coletados pelo Inventário COPE e os dados qualitativos obtidos nas entrevistas.

\section{Resultados e Discussão}

Inicialmente, serão descritas as estratégias de enfrentamento obtidas pelo Inventário COPE e analisados os estilos de coping dos cuidadores de acordo com a Teoria Disposicional do Coping de Carver et al. (Carver et al., 1989; Carver, \& Connor-Smith, 2010; Connor-Smith, \& Sheier, 2011). A seguir serão apresentados os resultados da entrevista, analisados com base na proposta teórica de Walsh (2005), articulando-se às análises de interpretação entre os domínios de resiliência familiar e as estratégias e estilos de coping identificados.

Para grande parte dos entrevistados, as estratégias de coping mais utilizadas, ou seja, aquelas com medianas acima de 12 que representavam um uso muito frequente ("muitas vezes") foram: Coping ativo; Reinterpretação positiva e crescimento; Retorno para a religiosidade; Planejamento; Supressão de atividades concomitantes; Coping moderado; e Aceitação (Figura). 


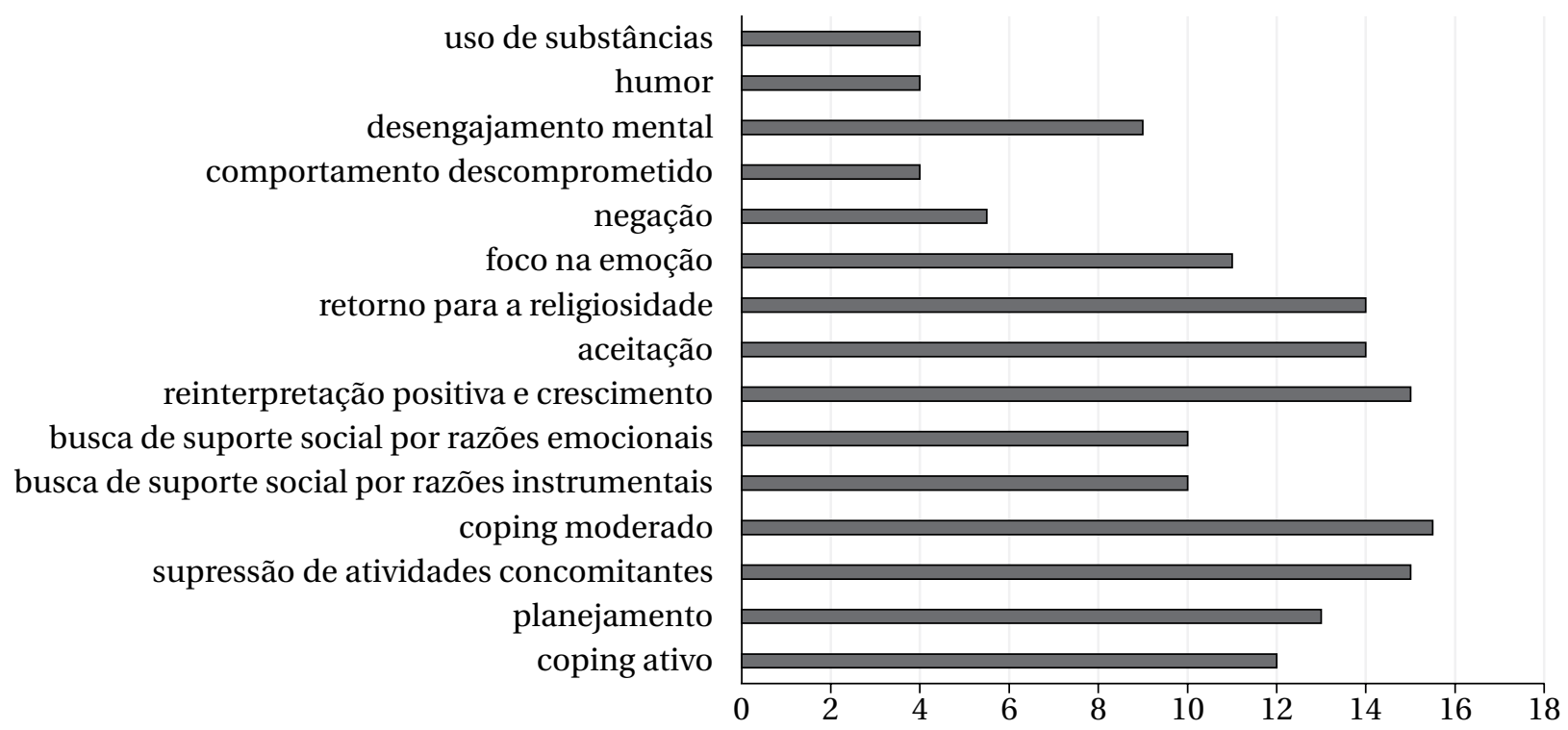

Figura

Estratégias de enfrentamento dos cuidadores pelo Inventário COPE $(\mathrm{N}=4)$.

Estratégias como Coping ativo, Planejamento, Supressão de atividades concomitantes e Coping moderado indicam que os cuidadores buscavam estabelecer passos sucessivos para remover, atenuar ou melhorar os efeitos do estressor, no caso a GTT. Isso fica claro também pelas entrevistas dos cuidadores, ilustrado pela seguinte fala: "meu esposo acabou arrumando dois empregos pra me deixar a inteira disposição dela. Dei baixa na carteira, saí do trabalho, não vou mais trabalhar para ter tempo integral com ela" (C4). Essas estratégias mais resolutivas de coping apareceram tanto na reorganização da família após o adoecimento do filho, como também no enfrentamento da GTT infantil, quando se observam relatos como: “Tudo eu pergunto, eles vê ai, ontem mesmo foi um dia de tensão de esperar e ela não poder fazer. Fui na ouvidoria, corri, tentei pelos meus meios vê se conseguia reverter. Eu luto. Me comunico bastante" (C3). Podem-se relacionar essas passagens, inclusive, às categorias "Processos organizacionais" e "Processos de comunicação" da resiliência familiar, que predizem que aquelas famílias se apresentavam flexíveis diante da crise que a GTT evoca. Frente a esta situação, os familiares demonstravam competências para mudar e se adaptar aos ajustes necessários diante do desafio representado pela cirurgia de GTT, o que representa um indicador de resiliência familiar importante para um bom funcionamento familiar (Walsh, 2005).
Com isso, essas famílias buscavam adotar passos concretos nesse momento de crise para uma resolução colaborativa dos problemas, com uma postura proativa e focada nos objetivos. De fato, por mais que a literatura considere que a GTT é uma indicação clínica que irá melhorar a qualidade de vida da criança (Anselmo et al., 2013; Santos et al., 2011; Souza, 2016), a decisão por este procedimento exigirá uma nova reorganização e funcionamento familiar para lidar com as mudanças na rotina da família após a cirurgia (Franco, 2016, p. 38), o que parece ter sido possível para aqueles cuidadores.

Já estratégias como Reinterpretação positiva $e$ crescimento e Aceitação representavam a capacidade dos cuidadores em reinterpretar o estressor de forma positiva e, desta forma, alcançar um crescimento pessoal com a situação de GTT. Este tipo de estratégia também indica que a família estava investida na aceitação deste procedimento como necessário para uma melhor condição de existência do filho, como se observa nesses relatos:

[...] ela é muito miúda por conta do coração que maltratou ela um tempo, por conta do respiratório que ela tem hoje e também pela opção de só comer leite. E a gastro, me falaram, que tem opção de entrar com outro tipo de comida (C4).

Ela vai colocar a gastro e vai pra fono pra fono incentivar ela a comer pela boca [...] Então ela 
sem a sonda ela vai. ela é muito esperta se você ensinar ela, ela pega logo. Então creio que vai ser pra melhorar isso ai, ela voltar a comer e o tratamento ter mais vantagem (C3).

Por estas falas percebe-se que os recursos de enfrentamento dos cuidadores se relacionavam a indicadores de resiliência familiar próprios do domínio "Sistema de crenças", com mudanças nas crenças familiares reconstruídas sob uma perspectiva positiva.

Com base em uma visão mais otimista da GTT, os cuidadores apresentavam-se confiantes na superação das dificuldades, como se pode observar nesse relato: "É chato, mas é uma coisa que não é pra toda vida, é provisória até ela melhorar e tal. Enfim, tem que saber né?! Aprender a lidar com isso" (C3). As crenças familiares em torno da GTT são aspectos importantes a serem considerados em estudos sobre resiliência familiar dessa natureza, já que, para algumas famílias, comer pela boca pode ser uma importante fonte de prazer e a privação disso representar o estigma de uma família anormal (Mahant et al., 2011), além da marginalização e discriminação dos filhos (Cruz et al., 2012). Logo, perceber que as crenças dos cuidadores em torno da GTT eram positivas indica um enfrentamento resiliente da situação.

De fato, foi majoritário para os cuidadores o uso de estratégias adaptativas próprias de um estilo de coping engajado, como o Copingativo e Planejamento, para lidar com as demandas da situação. A estratégia Planejamento, por exemplo, que foi uma das mais usadas, relaciona-se diretamente com a crença familiar sobre a necessidade do preparo da família para aprender sobre a GTT a fim de enfrentar, de forma resolutiva, o desafio que é conviver com uma criança ostomizada, como ilustra o relato: “[...] primeiro eu quero aprender, aqui ainda, como são os cuidados, como recebe a alimentação... Diante do que eu já vi aqui isso é um processo normal, não é nenhum 'bicho de sete cabeças'" (C4). Ainda que os pais tenham, em geral, medo de que os filhos sejam estigmatizados e discriminados pela sociedade (Cruz et al., 2012; Franco, 2016), os participantes deste estudo apresentavam-se otimistas e com expectativas positivas sobre a nova condição do filho. Certamente isso se deu pelo fato de que eles adotavam um estilo de coping engajado, no qual estratégias como Reinterpretação positiva e crescimento e Aceitação eram adotadas com base na construção de um significado positivo para situações de adversidade. Frente a um estressor, por hora interpretado como um desafio ao self, o uso de um estilo de coping engajado tem impacto resolutivo na superação das adversidades, como é a GTT infantil, pela capacidade de regular comportamentos e modular emoções para ultrapassar obstáculos (Carver et al., 1989; Carver, \& Connor-Smith, 2010; Carver, \& Scheier, 2011). Falas como "Fiquei pensando assim 'por quê?'. Aí eu vi que por ali vai ser melhor pra ela, pra alimentar porque até ela engolir pela boca, por onde ela vai se alimentar?" (C3) são exemplos disso.

Outra estratégia frequentemente usada pelos cuidadores foi o Retorno para a religiosidade. Mazon et al. (2008) e Silva, Girão, \& Cunha (2016) confirmam que é comum que cuidadores de crianças com deficiência se apoiem na fé religiosa para lidar com o impacto dessa condição, até mesmo no caso de pais. Também neste estudo os cuidadores se voltavam para a espiritualidade/religiosidade para lidar com a crise desencadeada pela tomada de decisão pela GTT, o que pode ser ilustrado a seguir: "Acho que Deus não dá... se ele achasse que eu não dava conta isso não tava acontecendo comigo... ela me consola de um lado. Não reclamo de tudo que eu tô passando, não culpo ninguém. Tem que acontecer mesmo" (C3). Outros relatos indicam, ainda, que o uso da fé religiosa esteve presente relacionando-se à regulação emocional proporcionada pela estratégia Religiosidade. Para Carver e Connor-Smith (2010), a Religiosidade pode representar uma estratégia de coping engajado quando a pessoa se sustenta na fé para obter consolo e se aceitar ativamente a situação, como se vê nessa fala: "Eu sou evangélica, eu divido com Deus isso. E aí troca, aí vem aquele sentimento que tudo vai dar certo porque Deus está permitindo essa situação" (C4). Por sua vez, a Religiosidade é uma estratégia de enfrentamento que dialoga com os aspectos de transcendência e espiritualidade do domínio "Sistema de crenças", já que, por meio dela, os cuidadores buscavam valores e propósitos amplos, baseados na fé ("A minha fé, eu tomo a decisão e não fico assim 'será que faço isso?'. Não... é minha força... Entrega na mão de Deus, que a gente vai prejudicar a menina se a gente ficar nessa de não deixar” (C1) e na transformação (“[...] ela mudou a minha vida... eu acho, eu penso que isso é uma coisa pra melhor, lá na frente" (C3) como meio de sobreviver à crise provocada pela GTT.

Ressalta-se que as estratégias nunca usadas pelos cuidadores foram: Uso de substâncias, Humor e Com- 
portamento descomprometido, todas características de um estilo de coping desengajado. Logo, os resultados indicam que os cuidadores apresentavam um estilo de coping engajado, porque usavam comportamentos ativos e atitudes resolutivas para o enfrentamento dos estressores. De acordo com a proposta da Teoria Disposicional do coping (Carver et al., 1989; Carver, \& Connor-Smith, 2010; Connor-Smith, \& Sheier, 2011), o enfrentamento daqueles cuidadores se basearia na interpretação de que a decisão pela GTT pode ser percebida e interpretada como um desafio a ser superado, o que pode ser assim ilustrado:

Com sonda lá eu virei uma enfermeira dela. Tanto que eu não fico receosa de mexer. Mas a parte da gastro eu vou aprender aqui, porque a gente tem um tempo pra lidar com a situação aqui pra quando eu for pra casa ficar mais focada (C3).

Carver e Connor-Smith (2010) consideram que o estilo de coping engajado facilita ao cuidador, no seu processo de enfrentamento, assumir uma atitude de compromisso e envolvimento para atenuar o estresse percebido, como se observa nesta fala: "Eu converso, vou na prefeitura, na secretaria de saúde. Aí fala assim 'tem que ir em outro hospital, avaliar outras coisas', aí no dia seguinte já tô no pé deles” (C2).

Em pesquisas sobre enfrentamento da deficiência pela família (Silva et al., 2016) é comum que os cuidadores adotem um coping focalizado no problema como modo de enfrentamento do diagnóstico do filho, indicando, assim, que as famílias buscam resolver seu conflito com a reelaboração do significado da situação estressora. Os dados deste estudo corroboram esta premissa, já que os participantes utilizaram como estratégia de enfrentamento a reinterpretação de que o estresse frente à decisão pela GTT pode ser superado por meio de uma atitude ativa de busca por novos significados e caminhos possíveis para lidar com a situação e resolver as demandas decorrentes: “[...] acho que tudo que acontece, você tem que passar por aquilo ali, lá na frente você vai... se eu não visse tudo que eu vi, ai o que ela vai passar, eu ia tá derrubada" (C3).

Pela análise das falas dos cuidadores pode-se perceber que o "Sistema de crenças" foi o domínio mais utilizado pelos familiares, visto que eles buscavam extrair significado da adversidade, com avaliação da crise e da angústia provocada pela GTT, como ilustra o relato: "[O que significa para você e sua família este momento de tomada de decisão pela gastrostomia?] Uma solução. Entendeu? Porque a gente já estava buscando há muito tempo, esperando essa busca pela solução. Então quando a gastro foi indicada significou uma solução" (C4). Conforme a proposta teórica de Walsh (2005), diante de uma crise, entender os acontecimentos desencadeados, conhecer as opções e as implicações desse evento estressor para toda a família é importante para o bem-estar familiar. Esta proposta teórica dialoga com os dados da entrevista "Indicadores de Resiliência Familiar", que indicaram que diante da crise os cuidadores buscaram extrair significado deste momento, mantiveram uma perspectiva positiva, apoiaram-se na fé, buscaram a colaboração da rede familiar e da equipe de saúde e conseguiram expressar seus medos, angústias e dúvidas diante dessa decisão. Logo, esse indicador de resiliência familiar pode ajudar a família a melhor enfrentar as demandas de uma criança ostomizada, tanto em relação aos cuidados pós-GTT, como em relação à reorganização psíquica da dinâmica familiar e seu enfrentamento dos estigmas socioculturais (Cruz et al., 2012; Franco, 2016; Mahant et al., 2016).

Embora seja comum cuidadores de crianças que serão ostomizadas experimentarem sentimentos de desapontamento e incapacidade de lidar com as demandas dos filhos, que são atualizados diante da tomada de decisão pela GTT (Cruz et al., 2012), nossos resultados revelaram que aqueles cuidadores empreendiam um processo de enfrentamento da GTT adotando uma perspectiva positiva marcada pela iniciativa ativa ("Ela falou 'vou assinar', eu falei 'também vou assinar'.. Tomei essa decisão confiante” - C1), e perseverança (“ $[. .$.$] a parte da gastro eu vou aprender$ aqui, porque a gente tem um tempo pra lidar com a situação aqui pra quando eu for pra casa ficar mais focada..." - C3). Ainda que essa tomada de decisão pela alimentação via gastrostomia seja um momento de incertezas e de grande conflito e estresse para os pais (Mahant et al., 2011), os cuidadores do estudo mantiveram a esperança, como pode ser observado no relato: "Porque eu vi lá no outro hospital essa parte de gastro. É chato, mas é uma coisa que não é pra toda vida, é provisória até ela melhorar e tal” (C3).

Para Franco (2016), a questão principal para as famílias no momento da decisão pela GTT se pauta na reorganização familiar depois da crise gerada pelo procedimento e pela perda da perspectiva de normalidade do filho, que reverbera nas suas próprias expectativas 
de uma vida normal e saudável. O estigma de deficiência que a GTT encerra resulta em questionamentos e insegurança para os pais, que podem lidar de forma mais resiliente com a situação quanto melhor for o engajamento deles no enfrentamento do problema.

Nesse sentido, indicando a reestruturação familiar diante da crise, outro domínio de resiliência familiar foi identificado no discurso dos cuidadores. Observou-se a presença de indicadores de resiliência familiar como os "Processos organizacionais", quando nas famílias daqueles cuidadores observava-se a competência para mudança: “[...] Acho que vai ser melhor, em vista da sonda, a gastro vai ficar melhor pra ela brincar, sabe?! [...] Aqui acho que vai ser uma outra etapa" (C3). Também foram observados apoio mútuo e liderança forte "Meu esposo vai muito no que eu decidir [...]" (C4); e a mobilização da família ampliada e da rede comunitária:

Pestalozi é o pai que leva, pra APAE é o carro da prefeitura, já no Rio é a secretaria de saúde que traz [...] As duas têm benefício [...] a médica me dá os remédio que preciso, tem um que o governo me dá, tem uma moça que me dá também [...] Negócio de compra também, todo quarto sábado do mês eles dão uma cesta básica pras minhas filhas (C2).

Como nos indica Cruz et al. (2012), a organização da família e o apoio da rede familiar e comunitária são fundamentais e influenciam na percepção dos cuidadores de que a cirurgia de GTT será bem-sucedida. Apesar de Mahant et al. (2011) indicarem que existem tensões instaladas pelo conflito entre as condições clínicas da criança e a representação simbólica da alimentação para a comunidade e núcleo familiar, pôde-se observar nesse estudo que a família ampliada e as redes comunitárias apareceram como um recurso social importante para a organização da família, como ilustra esse relato: "Tem minha família, o apoio deles. Tanto financeiramente quanto psicologicamente, meus irmãos são muito bons, minha mãe [...] Família muito unida, graças a Deus. Se não fosse taria tudo pior" (C3).

Outro indicador de resiliência familiar do domínio "Processos organizacionais" observado nos relatos dos cuidadores foi a expressão emocional aberta e compartilhamento de sentimentos (“[...] medo eu sei que eu tenho, de ter ela com outra expressão, como ela vai sentir essas coisas no corpo dela. Acho que a palavra que resume tudo é medo" - C4), além da responsabilidade pelos próprios sentimentos e comportamentos ("Eu fico triste, depressiva. Assim, nunca maltrato ela, sabe. Assim, sempre dou carinho, eu protejo ela bem. Mas a gente não fica bem" - C3). Estes aspectos da resiliência familiar mostram-se fundamentais no trabalho das equipes de saúde com as famílias de crianças com indicação para GTT, uma vez que a expressão de sentimentos facilita que os profissionais reconheçam as dificuldades das famílias. Por sua vez, isso também possibilita aos cuidadores se reestruturarem psiquicamente e se reorganizarem para lidar com a situação, tendo como objetivo serem pais amorosos e nutrientes (Franco, 2016).

Outro ponto importante foram os escassos recursos econômicos que os cuidadores dispunham, já que apenas duas famílias (C1 e C2) recebiam benefícios sociais. Diante deste cenário, os cuidadores mostraram-se capazes de se articularem buscando estabilidade e realizando mudanças a partir de uma atitude flexível. Pôde-se observar, ainda, que os cuidadores encontraram meios de lidar com as demandas necessárias de adaptação à GTT da criança, sobrevivendo ao momento de crise, conforme nesse relato:

[...] meu esposo acabou arrumando dois empregos pra me deixar a inteira disposição dela... dei baixa na carteira, saí do trabalho, não vou mais trabalhar para ter tempo integral com ela... a vida é cheia de coisas que acontecem. Estou pensando em mudar de casa, não pela gastro em si mas pra ela ter um espaço melhor (C4).

O último domínio de resiliência familiar analisado foram os "Processos de comunicação". Segundo Walsh (2005), o funcionamento familiar é mais resiliente quando os membros da família são competentes para expressarem suas ideias e tomarem atitudes frente às demandas de uma situação de crise. Foi possível observar um funcionamento familiar resiliente neste domínio por meio de relato como:

[...] a minha família, eles querem ela em casa, eles estão ciente que ela vai ir com essa cirurgia, com esse meio de se alimentar, de respirar. Não ficam muito animados com essa ideia, mas eles querem ela em casa, eles acreditam quando eu explico, das possibilidades, eles confiam no que eu falo (C4). 
Assim, foi possível observar na análise do discurso dos cuidadores, juntamente com estratégias de enfrentamento como a Aceitação, que eles pareciam, por meio de habilidades de competência comunicativa, utilizar mensagens claras, consistentes e sem informações ambíguas para lidar com a GTT com uma postura de aceitação ativa, quer seja com os profissionais de saúde ([E com a equipe, como você avalia sua comunicação?] "É muito boa, a gente tá sempre conversando. Quando tenho dúvida eu tô sempre esclarecendo" - C4), quer seja com a família ("Todo mundo sabe o que vai acontecer, minha mãe já aceitou, meus irmãos também" - C2). Esse é um indicador de resiliência familiar que favorece a aceitação da GTT pelos cuidadores e, consequentemente, o envolvimento da família para um melhor prognóstico dos pacientes no momento pós-cirúrgico (Martínez-Costa et al., 2011; Schweitzer et al., 2014).

Segundo Walsh (2005), ao incentivar a resiliência familiar frente a doenças graves e crônicas, a equipe de saúde deve ser flexível para moldar suas intervenções quando necessário, assim como, saber lidar com os diferentes membros da família conforme suas particularidades. A autora aponta ainda que devem ser estabelecidas prioridades clínicas na intervenção com famílias que enfrentam doenças graves e crônicas. Tais intervenções devem se voltar para redução do impacto estressante da doença na família, proporcionando informações e oferecendo diretrizes práticas para lidar com as demandas do adoecimento durante as diferentes fases da doença. Importante, por fim, proporcionar vínculos com serviços que possam dar apoio aos esforços de cuidado da família e manter o funcionamento do paciente na comunidade (Walsh, 2005).

Portanto, o posicionamento da equipe de saúde, enquanto facilitador do enfrentamento e da resiliência familiar frente à GTT, deve se pautar na construção de uma relação equipe - cuidadores que possibilite a comunicação e a troca de informações, sem perder de vista a necessária flexibilidade para a manutenção de um bom funcionamento familiar e da equipe e sua assistência ao paciente. Ao indicar seus valores, os cuidadores permitem que a equipe trabalhe de forma articulada com a família, desempenhando, assim, um papel facilitador na tomada de decisão, por meio do atendimento baseado em evidências clínicas e integrado aos valores familiares. Esta é uma proposta de intervenção com maior eficácia na atenção a famílias candidatas à GTT, porque se baseia na empatia da equipe para com a família, além de protocolos assistenciais baseados na educação, informação e suporte de alta qualidade (Franco, 2016; Mahant et al., 2011; Martínez-Costa et al., 2011; Shelton, \& Jackson, 2011; Pemberton et al., 2013; Schweitzer et al., 2014).

Tal como na instituição em que o estudo foi realizado, não se tem registro de estudos nacionais sobre propostas de intervenção familiar para cuidadores de crianças candidatas à GTT. A estratégia SDM, sugerida por Nelson e Mahant (2014), parece ser uma intervenção eficaz também para promover a resiliência e competências da família para sua adaptação e enfrentamento da GTT, pois se propõe a adotar um enfoque sistêmico e não individual, próprio de trabalhos sobre resiliência familiar (Yunes, 2003).

Nesse sentido, profissionais de saúde também são parte integrante de uma perspectiva sistêmica de intervenção para promoção da resiliência e enfrentamento familiar frente ao adoecimento crônico da criança e às demandas da GTT. A equipe de saúde deve ser a principal apoiadora e fonte de informações para os cuidadores na tomada de decisão pela GTT e para tal ela deve ser capacitada para facilitar os processos organizacionais e comunicativos que favoreçam a resiliência familiar. Por meio da troca de informações e o esclarecimento das dúvidas a equipe pode, ainda, favorecer mudanças no sistema de crenças proporcionando a construção de uma perspectiva mais positiva da GTT. Isto é muito importante porque, segundo Susin et al. (2012), os cuidadores aceitam melhor a cirurgia de GTT quando existe um trabalho multidisciplinar que ofereça informações e orientações de qualidade.

Neste cenário em que cuidadores e equipe de saúde empreendem uma luta por uma melhor qualidade de vida da criança com necessidades especiais de saúde, coloca-se um dilema que deve ser superado com o esforço mútuo de todos: a decisão ou não pela GTT. Podemos concluir que as famílias são capazes de se envolver em um processo resiliente de enfrentamento dos desafios impostos pela GTT infantil e lidar com a adversidade de forma ativa e planejada, quando reinterpretam a experiência vivenciada e atribuem um significado positivo à situação, apoiando-se por vezes na religiosidade e na sua rede familiar e comunitária. Para facilitar esse processo, os profissionais de saúde podem ajudar os cuidadores quando são fonte de suporte emocional e informacional, oferecendo intervenções que promovam a resiliência familiar 
para superar o impacto psicológico da GTT infantil. Para os cuidadores deste estudo, que adotaram um estilo de coping engajado com prevalência de estratégias como Reinterpretação positiva e crescimento e Aceitação, associado aos indicadores de resiliência familiar observados, pode-se sugerir que a atenção à saúde sistêmica, oferecida à criança e sua família, disponibilizou um funcionamento familiar resiliente.

\section{Conclusão}

Diante do desafio de tomar uma decisão pela gastrostomia dos filhos, os cuidadores conseguiram atribuir novos significados à adversidade, utilizando-se da espiritualidade, da atitude ativa, da perseverança, do enfrentamento do possível e da aceitação do que não poderia ser mudado. Merece destaque o papel da equipe de saúde neste momento de tomada de decisão, considerada pelos cuidadores como um apoio importante e necessário para a reestruturação da organização familiar. A equipe de saúde deve atuar como facilitadora por meio de estratégias de negociação com os cuidadores, fornecendo informações e esclarecendo suas dúvidas. Cabe ressaltar que essa negociação envolveu expor e aceitar as dúvidas e críticas dos cuidadores a fim de buscar objetivos compartilhados entre eles, a equipe e as necessidades de saúde da criança. Portanto, a tomada de decisão pela GTT foi pautada, tanto nos valores familiares e senti- mentos dos cuidadores, como também no papel que a equipe desempenha como fonte de apoio social e emocional, cujo saber clínico é necessário à situação. Logo, conclui-se que nessa tomada de decisão é fundamental oferecer um olhar e uma escuta clínica dos sentimentos, crenças e valores dos cuidadores. Com base nessa atitude empática para com a família, a equipe de saúde deve privilegiar o cuidado ao cuidador, incentivando uma tomada de decisão compartilhada, sem posições rígidas e baseada em um diálogo compreensivo, paciente e sem críticas.

Considera-se como limitações do estudo o número restrito de entrevistados, que dificulta a generalização dos resultados e, por conseguinte, a análise de variáveis importantes do enfrentamento e da resiliência familiar, como o suporte financeiro, por exemplo. Certamente, as demandas financeiras próprias do cuidado de uma criança com GTT agregam dificuldades no enfrentamento da tomada de decisão por este procedimento. Por ser uma situação de grande exigência financeira, dificuldades de acesso às políticas públicas e uma análise mais acurada dos motivos pelos quais as mães (principais cuidadoras) não trabalhavam e as consequências disso poderiam ampliar a avaliação das nuances do enfrentamento e suas repercussões para resiliência familiar na situação de GTT, que é uma condição de vulnerabilidade física e psicológica tanto para a criança como para o cuidador.

\section{Referências}

Anselmo, C. B., Tercioti Junior, V., Lopes, L. R., Coelho Neto, J. S., \& Andreollo, N. A. (2013). Gastrostomia cirúrgica: Indicações atuais e complicações em pacientes de um hospital universitário. Revista do Colégio Brasileiro de Cirurgiões, 40(6), 458-462. https://doi.org/10.1590/S0100-69912013000600007

Antoniazzi, A. S., Dell'Aglio, D. D., \& Bandeira, D. R. (1998). O conceito de coping: Uma revisão teórica. Estudos de Psicologia (Natal), 3(2), 273-294. https://doi.org/10.1590/S1413-294X1998000200006

Avitsland, T. L., Faugli, A., Pripp, A. H., Malt, U. F., Bjørnland, K., \& Emblem, R. (2012). Maternal psychological distress and parenting stress after gastrostomy placement in children. Journal of Pediatric Gastroenterology \& Nutrition, 55(5), 562-6. https://doi.org/10.1097/MPG.0b013e31826078bd

Bardin, L. (2006) Análise de conteúdo. Lisboa: 70.

Brasil. Ministério da Saúde. (2013). Diretrizes de atenção à pessoa com paralisia cerebral. Brasília, DF: o autor. Recuperado de: http://bvsms.saude.gov.br/bvs/publicacoes/diretrizes_atencao_paralisia_cerebral.pdf

Carver, C. S., \& Scheier, M. F. (2011). Self regulation of action and affect. In K. D. Vohls, \& R. F. Baumeister, Handbook of self regulation: Research, theory and applications (2a ed., pp. 3-21). New York, NY: Guilford.

Carver, C. S., \& Connor-Smith, J. (2010). Personality and coping. Annual Review of Psychology, 61, 679-704. https:// doi.org/10.1146/annurev.psych.093008.100352

Carver, C. S., Scheier, M. F., \& Weintraub, J. K. (1989). Assessing coping strategies: A theoretically based approach. Journal of Personality and Social Psychology, 56(2), 267-83. https://doi.org/10.1037/0022-3514.56.2.267 
Cravinho, C. R. M., \& Cunha, A. C. B. (2015). Enfrentamento da morte fetal pela enfermagem na abordagem disposicional e na teoria motivacional do coping. Estudos de Psicologia (Campinas), 32(2), 307-317. https://doi. org/10.1590/0103-166X2015000200014

Cruz, A. C., Angelo, M., \& Gamboa, S. G. (2012). A visão da família sobre a experiência de ter uma criança gastrostomizada. Revista de Enfermagem Referência, 3(8), 147-153. https://doi.org/10.12707/RIII1216

Franco, V. (2016) Tornar-se pai/mãe de uma criança com transtornos graves do desenvolvimento. Educar em Revista, (59), 35-48. https://doi.org/10.1590/0104-4060.44689

Lazarus, R. S., \& Folkman, S. (1984). Stress, appraisal and coping. New York, NY: Springer.

Mahant, S., Jovcevska, V., \& Cohen, E. (2011). Decision-making around gastrostomy-feeding in children with neurologic disabilities. Pediatrics, 127(6), 1471-1481. https://doi.org/10.1542/peds.2010-3007

Martínez-Costa, C., Borraz, S., Benlloch, C., López-Sáiz, A., Sanchiz, V., \& Brines, J. (2011). Early decision of gastrostomy tube insertion in children with severe developmental disability: A current dilemma. Journal of Human Nutrition and Dietetics, 24(2), 115-21. https://doi.org/10.1111/j.1365-277X.2010.01146.x

Martínez-Costa, C., Calderón, C., Pedrón-Giner, C., Borraz, S., \& Gómez-López, L. (2013). Psychometric properties of the structured satisfaction questionnaire with gastrostomy feeding (SAGA-8) for caregivers of children with gastrostomy tube nutritional support. Journal of Human Nutrition and Dietetics, 26(2), 191-197.https://doi.org/10.1111/jhn.12000

Martins, A. C. F. (2013). Perfil de pacientes portadores de gastrostomia e o papel dos cuidadores no domicílio (Dissertação de mestrado). Faculdade de Medicina, Universidade Estadual Paulista Júlio de Mesquita Filho, Botucatu, São Paulo, Brasil. Recuperado de http://hdl.handle.net/11449/108609

Mazon, V., Carlotto, M. S., \& Câmara, S. (2008). Síndrome de burnout e estratégias de enfrentamento em professores. Arquivos Brasileiros de Psicologia, 60(1), 55-66.

Nelson, K. E., \& Mahant, S. (2014). Shared decision-making about assistive technology for the child with severe neurologic impairment. Pediatric Clinics of North America, 61(4), 641-52. https://doi.org/10.1016/j.pcl.2014.04.001

Noronha, M. G. R. C. S., Cardoso, P. S., Moraes, T. N. P., \& Centa, M. L. (2009). Resiliência: Nova perspectiva na promoção da saúde da família? Ciência \& Saúde Coletiva, 14(2), 497-506. https://doi.org/10.1590/S141381232009000200018

Pemberton, J., Frankfurter, C., Bailey, K., Jones, L., \& Walton, J. M. (2013). Gastrostomy matters: The impact of pediatric surgery on caregiver quality of life. Journal of Pediatric Surgery, 48(5), 963-70. https://doi.org/10.1016/j. jpedsurg.2013.02.012

Portaria $N^{o}$ 400, de 16 de novembro de 2009. Estabelece diretrizes nacionais para a Atenção à Saúde das pessoas ostomizadas no âmbito do Sistema Único de Saúde - SUS. Recuperado de http://bvsms.saude.gov.br/bvs/saudelegis/sas/2009/prt0400_16_11_2009.html

Santos, J. S., Kemp, R., Sankarankutty, A. K., Salgado, W., Jr., Tirapelli, L. F., \& Silva, O. C., Jr. (2011). Gastrostomia e jejunostomia: Aspectos da evolução técnica e da ampliação das indicações. Medicina, 44(1), 39-50.

Schweitzer, M., Aucoin, J., Docherty, S. L., Rice, H. E., Thompson, J., \& Sullivan, D. T. (2014). Evaluation of a discharge education protocol for pediatric patients with gastrostomy tubes. Journal of Pediatric Health Care, 28(5), 420-8. https:// doi.org/10.1016/j.pedhc.2014.01.002

Shelton, J., \& Jackson, G. P. (2011). Palliative care and pediatric surgery. The Surgical Clinics of North America, 91(2), 419-28. https://doi.org/10.1016/j.suc.2011.01.004

Silva, E. H. P., Girao, E. R. C., \& Cunha, A. C. B. (2016). Enfrentamento do pai frente à malformação congênita do filho antes e depois do nascimento. Estudos e Pesquisa em Psicologia, 16(1), 180-199.

Souza, E. C. (2016). Uma nova técnica de gastrostomia cirúrgica a partir de conceitos da gastrostomia endoscópica. ABCD: Arquivos Brasileiros de Cirurgia Digestiva, 29(1), 50-52. https://doi.org/10.1590/0102-6720201600010013

Souza, M. T. S., \& Cerveny, C. M. O. (2006). Resiliência psicológica: Revisão da literatura e análise da produção científica. Revista Interamericana de Psicologia, 40(1), 119-126.

Susin, F. P., Bortolini, V., Sukiennik, R., Mancopes, R., \& Barbosa, L. R. (2012). Perfil de pacientes com paralisia cerebral em uso de gastrostomia e efeito nos cuidadores. Revista CEFAC, 14(5), 933-942. https://doi.org/10.1590/ S1516-18462012005000016 
Walsh, F. (2005). Fortalecendo a resiliência familiar. São Paulo, SP: Roca.

Wilson, M., Gosche, J., Bishop, P., Liu, H., Moore, T., \& Nowicki, M. J. (2010). Critical analysis of caregiver perceptions regarding gastrostomy tube placement. Pediatrics International, 52(1), 20-5. https://doi.org/10.1111/j. 1442-200X.2009.02851.X

Yunes, M. A. M. (2003). Psicologia positiva e resiliência: O foco no indivíduo e na família. Psicologia em Estudo, 8(esp), 75-84. https://doi.org/10.1590/S1413-73722003000300010

\section{Natália Ferraz Novaes}

Psicóloga residente na Residência Multiprofissional em Saúde da Criança e do Adolescente no Instituto de Puericultura e Pediatria Martagão Gesteira da Universidade Federal do Rio de Janeiro (UFRJ), Rio de Janeiro - RJ. Brasil.

E-mail: novaespsi@hotmail.com

(iD) https://orcid.org/0000-0003-2425-6177

\section{Ana Cristina Barros Cunha}

Professora Associada do Departamento de Psicologia Clínica, Instituto de Psicologia, da Universidade Federal do Rio de Janeiro (UFRJ), Rio de Janeiro - RJ. Brasil.

E-mail: acbcunha@yahoo.com.br

(iD) https://orcid.org/0000-0003-0839-0130

Endereço para envio de correspondência:

Avenida Marquês de Paraná, 349, bloco 1, apt 1209, Centro. CEP: 24030-215. Niterói-RJ. Brasil.

Recebido 09/11/2017

Reformulado 16/10/2018-18/12/2018

Aceito 08/01/2019

Received 11/09/2017

Reformulated 10/16/2018-12/18/2018

Approved 01/08/2019

Recibido 09/11/2017

Reformulado 16/10/2018 - 18/12/2018

Aceptado 08/01/2019

Como citar: Novaes, N. F., \& Cunha, A. C. (2019) Enfrentamento e Resiliência Familiar na Tomada de Decisão pela Gastrostomia Infantil. Psicologia: Ciência e Profissão 2019 v. 39, 1-16. https://doi.org/10.1590/1982-3703003187678

How to cite: Novaes, N. F. \& Cunha, A. C. (2019) Coping and Family Resilience in Decision-Making about Children's Gastrostomy. Psicologia: Ciência e Profissão 2019 v. 39, 1-16. https://doi.org/10.1590/1982-3703003187678

Como citar: Novaes, N. F, \& Cunha, A. C. (2019) Enfrentamiento y Resistencia en la Toma de Decisiones por Gastrostomía para Niños. Psicologia: Ciência e Profissão 2019 v. 39, 1-16. https://doi.org/10.1590/1982-3703003187678 